\title{
Probing viral assembly
}

Drugs that interfere with viral capsid assembly hold potential as antiviral agents but have proven difficult to identify by conventional screening methods. In Nature Biotechnology, Adam Zlotnick and colleagues describe a new technique for monitoring the capsid assembly process, which might aid the identification of such compounds.

Assembly of the viral coat, or capsid, is an integral step in the viral replication cycle, making it a prospective therapeutic target in acute and chronic viral infections. The proteins that make up the capsid are unique to the virus and distinct from host cellular proteins, which gives drugs that target assembly a reduced likelihood of toxicity. However, this approach remains underexploited, in part because of the lack of suitable screening methods for assembly inhibitors. Now researchers have developed a rapid and sensitive technique for monitoring hepatitis $B$ virus (HBV) capsid assembly in vitro.

To detect capsid assembly, distance-sensitive fluorescent probes were attached to the $\mathrm{C}$ termini of $\mathrm{HBV}$ capsid protein assembly domains. During in vitro assembly, the labelled $\mathrm{C}$ termini are brought closer together on the inner surface of the capsid, resulting in self-quenching between dye molecules and a reduction in emitted fluorescence. Compounds that interfere with the assembly process therefore produce a measurable change in fluorescence quenching.
The technique was applied to two agents that are known to alter capsid assembly: HAP1 and urea. HAP1 causes accelerated and misdirected assembly and resulted in an increase in fluorescence quenching. In contrast, urea inhibits capsid assembly and was shown to decrease the degree of quenching. The technique therefore allows the detection of both compounds that inhibit assembly and those which act by misdirecting the process. Furthermore, by testing lower concentrations of these agents, it was shown that even relatively subtle effects on capsid assembly can be successfully detected by this assay.

The assay could potentially be scaled up to allow high-throughput screening of compounds that disrupt

\section{ANTICANCER DRUGS}

\section{Targeting resistance}

Most cancer cells acquire resistance to apoptosis-inducing drugs such as the widely-used drug doxorubicin. Brent Stockwell and colleagues used highthroughput cell-viability screening and a pathway-based analysis to find compounds that increase the lethality of doxorubicin in cancer cells that are resistant to it.

Two colon carcinoma cell lines were used for the screens. One cell line (RKO) expressed high levels of the DNA damage-response protein $\mathrm{p} 53$ and was sensitive to doxorubicin (a topoisomerase-II-based DNA-damaging agent). The other cell line (RKO-E6) expressed the viral oncoprotein $\mathrm{E} 6$, which induces $\mathrm{p} 53$ degradation, and was relatively resistant to doxorubicin. The authors reasoned that compounds that overcome E6-induced resistance should restore doxorubicin's lethality in RKO-E6 cells. Nearly 30,000 compounds were tested in the primary screen, and 278 of these were lethal. A secondary screen eliminated those compounds that did not depend on E6 and doxorubicin to induce cell death -88 compounds remained.
So, what is the basis of the resensitization activity of these 88 compounds? Surprisingly, only one compound affected the expression level of $\mathrm{p} 53$, so the authors developed a pathway-based analysis using a co-treatment strategy to determine how the compounds functioned. In this analysis, compounds that increased the sensitivity of RKOE6 cells to the microtubule inhibitor podophyllotoxin, the topoisomeraseI-based DNA-damaging agent camptothecin, and doxorubicin were assumed to act through general celldeath mechanisms and were eliminated. Compounds that increased sensitivity to both camptothecin and doxorubicin but not podophyllotoxin presumably act downstream of the DNA-damage response. Compounds that synergized only with doxorubicin were likely to operate at the level of, or upstream of, topoisomerase Il proteins. These last two groups of compounds were studied in more detail.

The authors found several groups of compounds with the potential to restore doxorubicin lethality. These included quaternary ammonium compounds, protein-synthesis inhibitors, and a class of small molecules that included 1,3-bis(4-morpholinymethyl)-2-imidazolidinethione. The authors named another previously unexplored class of small molecules indoxins, for their ability to increase doxorubicin's lethality selectively. All of these classes of compounds upregulated topoisomerase Il $\alpha$ and/or induced S-phase arrest in RKO-E6 cells.

To further elucidate the mechanism of action of the indoxins, the authors synthesized a series of indoxin analogues and used indoxin affinity probes to identify their protein targets. One of the five proteins identified was myosin 1c. In keeping with its known functions, it is possible that nuclear myosin 1c mediates topoisomerase Il $\alpha$ transcription, whereas myosin $1 \mathrm{c}$ in the cytosol might mediate S-phase arrest.

Further investigation of these doxorubicin-enhancing small molecules for the adjuvant treatment of doxorubicinresistant cancer is warranted.

Ezzie Hutchinson, Nature Reviews Cancer

ORIGINAL RESEARCH PAPER Smukste, I., Bhalala,

O., Perisco, M. and Stockwell, B. R. Using small molecules to overcome drug resistance induced by a viral oncogene. Cancer Cell 9, 133-146 (2006)
Stockwell and colleagues used highthroughput cell-viability screening and a pathwaybased analysis to find compounds that increase the lethality of doxorubicin. go 
assembly. The use of fluorescent dyes can overcome technical difficulties associated with other assembly assays, such as light scattering, which is more sensitive to the presence of particulates or aggregates in the solution. Furthermore, this technique is both faster and cheaper than cell-based antiviral assays and is not complicated by issues associated with cellular uptake or toxicity of the candidate drug. At present, HBV capsid assembly is one of the better understood systems; however, as we gain improved understanding of viral assembly this approach could translate to other viruses that rely on capsid assembly for replication, including HIV, hepatitis $\mathrm{C}$ and avian influenza.

Katherine Whalley

ORIGINAL RESEARCH PAPER Stray, et al. An in vitro fluorescence screen to identify antivirals that disrupt hepatitis B virus capsid assembly. Nature Biotechnol. 24, 358-362 (2006)

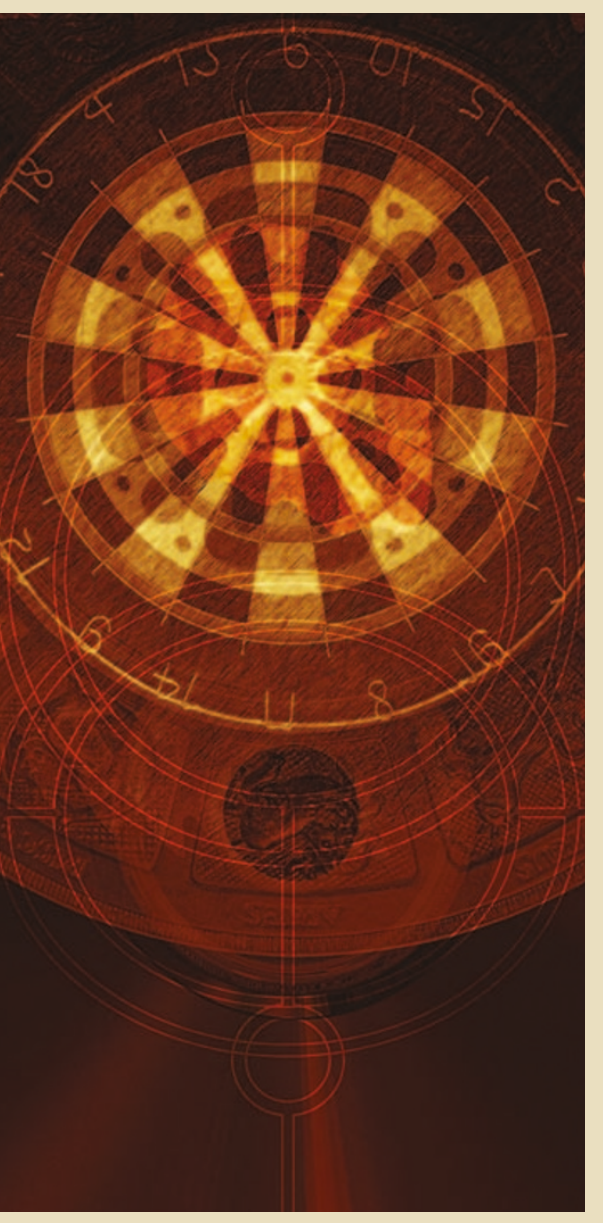

\section{Parallel lines}

Screening of compound libraries against cells is usually limited to characterizing the effects of molecules against a single cell line or drug target. The ability to study the effects of a compound on various cellular parameters simultaneously in different cell types could therefore speed up screening and enable parallel studies of drug efficacy and toxicity. Jeremy Caldwell and colleagues describe in Proceedings of the National Academy of Sciences a robotic system that does just that, and demonstrate how this system was used to characterize a library of kinase inhibitors and identify novel activities for known kinase inhibitors that could broaden their therapeutic use.

The authors designed an automated compound profiling (ACP) system that enables automated cell culture and which can perform miniaturized cell-based assays in 384- or 1,536-well microplates. The system integrates all stages of cell-line propagation (passaging, splitting, and determination of cell density and viability) with the necessary steps for compound screening (cell dispensing, compound addition, incubation, detection, reagent addition and plate reading). The authors demonstrated the capability of the ACP system by profiling the growth-inhibitory properties of a library of kinase-directed heterocyclic compounds against a panel of cells constitutively expressing a specific tyrosine kinase.

Kinases are often constitutively activated by fusion with other genes, and so Caldwell and colleagues exploited this to generate a cDNA library of tyrosine kinases fused to a common kinase fusion partner, ETV6/Tel, which, when expressed in a haematopoietic cell line, conferred tyrosine-kinase-dependence on cell populations. The library of tyrosine kinase-targeted compounds was supplemented with 10 known tyrosine kinase inhibitors and screened against the kinase-dependent cells. Only 30 of the 1,400 compounds were toxic to the cells, and 282 compounds had no effect on any kinasedependent cell line. However, each kinase tested was inhibited by at least one small molecule in the library, and $5.9 \%$ of the compound library selectively inhibited a single kinase.

Clusters of structurally similar molecules showed a modest correlation between potency and selectivity (as is typically identified by conventional kinase screens), and a plot of chemical similarity versus growth inhibition for pairs of compounds suggested that chemical similarity was in most cases an effective predictor of biological activity. Moreover, structure-activity relationship dendrograms compiled from the ACP data confirmed that structurally homologous kinases had comparable inhibition profiles, and could be used to aid target selection.

Perhaps the most therapeutically significant finding was the previously uncharacterized activities of existing kinase inhibitors. In addition to confirming the cross-kinase effects of imatinib (Gleevec; Novartis) on BCR-ABL, platelet-derived growth factor and c-KIT, new activities for less well-characterized kinase inhibitors currently in development were observed. BIRB796, a p38 kinase inhibitor, was also found to inhibit TIE1 and TIE2, kinases involved in angiogenesis and stem-cell quiescence and mobilization during chemotherapy. BMS-354825, a dual SRC and ABL inhibitor currently in trials as a therapy to overcome imatinib-resistant acute lymphoblastic and chronic myelogenous leukaemia, also inhibited multiple additional kinases including the ephrin receptors $\mathrm{B} 1, \mathrm{~B} 2$ and $\mathrm{B} 4$.

Discovering these activities early on in screening not only highlights the potential for expanding investigative drugs for other indications, but could perhaps also explain off-target effects. Although others have profiled the 'kinome' against chemical space, this paper is the first example of the use of a physiologically relevant cell-based kinase screen used in parallel with a large compound library, and could be adapted to screen other target and drug classes.

Joanna Owens

ORIGINAL RESEARCH PAPER Melnick, J. S. et al. An efficient rapid system for profiling the cellular activities of molecular libraries. Proc. Natl Acad. Sci. USA 103, 3153-3158 (2006) WEB SITE

Jeremy S. Caldwell's laboratory:

http://web.gnf.org/scientific/cellbio/caldwell.html

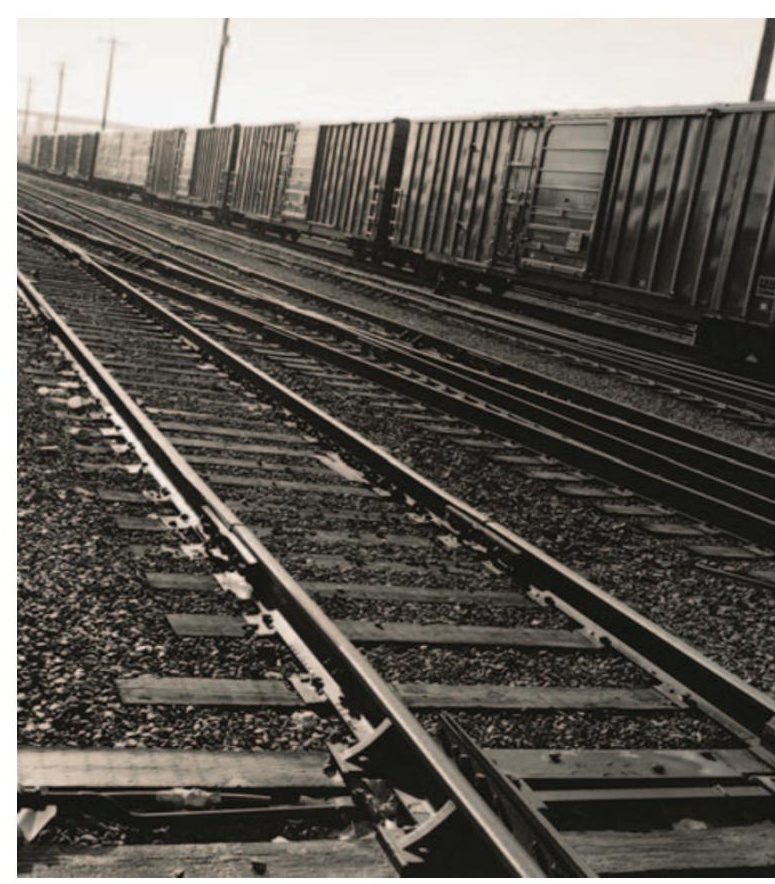

